\title{
Simple holographic polarogram
}

\author{
Maria José Inestrosa, Hernán Chuaqui, ${ }^{*}$ Raúl Aliaga-Rossel, Mario Favre, \\ lan Mitchell, and Edmund Wyndham \\ Pontificia Universidad Católica de Chile, Avenida Vicuña Mackenna 4860, Macul, Santiago 782-0436, Chile \\ *Corresponding author: hchuaqui@fis.puc.cl
}

Received 11 September 2007; revised 2 December 2007; accepted 22 January 2008; posted 23 January 2008 (Doc. ID 87347); published 28 February 2008

\begin{abstract}
A new technique capable of obtaining quantitative values of the rotation angle of the polarization vector by using holography is presented. This is a two-stage holographic process; during the recording stage a hologram of the object of interest is obtained. The reference beam is composed of two beams that form a small angle between them and keep their polarization states at right angles to each other. In the reconstruction stage of the hologram, two images from the hologram are obtained along two different angles. As a result of the interference between these two images, a set of parallel fringes is formed at the image plane. The fringe contrast on the reconstruction is related to the angle of the polarization vector of the light at each position on the image plane. Measurements of the rotation of the polarization angle of a fraction of a degree were obtained. The main application of this technique is in the study of transient phenomena, where single-shot measurements are the only means of obtaining reliable data. (C) 2008 Optical Society of America

OCIS codes: $\quad 030.0030,000.2170,120.2130$.
\end{abstract}

\section{Introduction}

Holography has been described as capable of recording all the information carried by a wavefront. However, in a normal holographic setup the information about the state of polarization of light is lost. Several schemes to record the polarization state have been proposed, such as the use of two separate reference beams [1,2], or the use of a mosaic polarizer [3]. A separate two-reference-beam scheme for metal surfaces was proposed by Garvanska [4]. These methods use standard silver halide holographic emulsions as the recording media. A different approach is to develop a polarization-sensitive emulsion [5]. The majority of available techniques employed in the measurement of polarization rotation of light use intensity variation through an analyzing polarizer. The main problem associated with this approach is the requirement for an absolute intensity measurement; therefore any unbalanced stray light does affect the measurement. This is particularly difficult in situations in which small-angle polarization rotation is

0003-6935/08/070883-05\$15.00/0

(C) 2008 Optical Society of America being considered. A method that to a large extent avoids some of the problems is given in [6], although it requires a succession of three images to obtain the polarization information and therefore is not suitable for single-shot events. A technique that could be used for single-shot events is Savart plates [7], which consist of two polarizing beam splitting plates with orthogonal optic axes. An incident beam propagating through plate 1 is resolved into ordinary and extraordinary beams, which are displaced from each other in the first principal plane. Upon entering plate 2 , the ordinary beam becomes an extraordinary beam, and vice versa. Splitting again takes place in the second principal plane, which is orthogonal to the first. The result is two emerging beams displaced along a diagonal. A set of interference fringes is obtained, in which the separation of the fringes is proportional to the plate thickness and the contrast is a measure of the polarization angle. The main problem of this method is that stray light does affect the fringe contrast measurement.

In this paper we present a method to obtain the polarization state of an arbitrary wavefront. This is achieved in a two-stage process. Two holograms 
are recorded on the same holographic plate. The only difference between the two holograms is that their reference beams are at a small angle with respect to each other and each has a different state of po>larization. The two reference beams are linearly polarized, and their axes are at right angles to each other. The dual hologram is reconstructed by using a single beam, giving rise to two reconstructed wavefronts bearing the same angle between them as the two reference beams. The reconstructed wavefronts interfere, generating a set of parallel fringes. From the fringe contrast the polarization state of different points of the wavefront are obtained. The method is suitable for phase or reflective objects. It can be applied to steady-state or transient situations.

\section{Theoretical Background and Experimental Setup}

The experimental configuration, shown in Fig. 1 , consists of a Nd:YAG CW frequency-doubled laser (532 nm, single longitudinal mode, $20 \mathrm{~mW}$ ). The laser beam is separated into two beams; one is used as reference and the other as object beam. In the path of each beam a half-wave plate (not shown) is located in order to control the polarization state of each beam. In the reference beam path a $0^{\circ} 40^{\prime}$ polarizing wedge is located. The wedge generates two reference beams with orthogonal polarizations and with an angular separation of $0.01 \mathrm{rad}$. One of the resulting reference beams polarization is parallel to the plane of the optical table, and the other's is perpendicular to it. The object beam polarization is rotated by $10^{\circ}$ relative to the polarization of one of the reference beams to generate a contrast bias.

The test object consists of a half-wave plate that covers only half of the field of view; the other half is left clear. Therefore the object consists of two adjacent areas of different polarizations; the polarization of the area in which the half-wave plate is

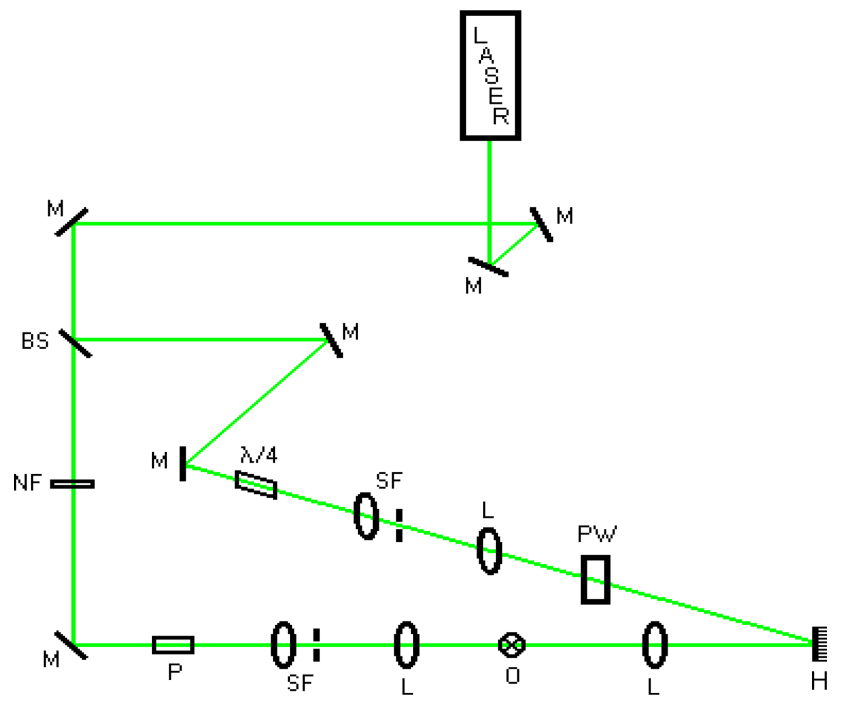

Fig. 1. (Color online) Experimental setup. M, mirror; BS, beam splitter; NF, neutral filter; L, lens; $\lambda / 4$, quarter-wave plate; $\mathrm{P}$, polarizer; $\mathrm{SF}$, spatial filter; $\mathrm{O}$, object; $\mathrm{PW}$, polarizing wedge; $\mathrm{H}$, holographic plate. located can be modified by rotating the half wave plate. Image plane holograms, as well as Fresnel holograms, were made in this set of experiments. During the recording stage, two holograms, one for each polarization component of the object beam, are formed on the same holographic emulsion. After development, a single beam is used to reconstruct the dual hologram, generating two reconstructed beams (one from each hologram obtained from the two reference beams). The two reconstructed beams provide two wavefronts from the same object, albeit at a slight angle given by the polarizing wedge. The reconstructed wavefronts correspond to the two polarization states of the object. The two reconstructed beams have the same polarization state, as they are obtained from the same illuminating beam. Therefore a set of parallel interference fringes is obtained. The fringe separation is controlled by the angle of the polarizing wedge.

We will use Jones matrix calculus to describe our setup. The Jones vector for a $45^{\circ}$ linearly polarized beam is given by

$$
\mathbf{R}_{+45^{\circ}}=\frac{1}{\sqrt{2}}\left(\begin{array}{l}
1 \\
0
\end{array}\right),
$$

and the Jones matrix for a polarizing wedge is given by

$$
\mathbf{J}_{\mathbf{P W}}=\left(\begin{array}{cc}
e^{-i \omega_{\|} x} & 0 \\
0 & e^{-i \omega_{\perp} x}
\end{array}\right)
$$

where $\omega_{\|}=\left(2 \pi \times \sin \theta_{\|}\right) / \lambda$ and $\omega_{\perp}=\left(2 \pi \times \sin \theta_{\perp}\right) / \lambda$; $\theta_{\|}$and $\theta_{\perp}$ are the beam angles of the parallel and perpendicular polarization components relative to the holographic plate normal.

In our experiment the reference beam is obtained by shining a $45^{\circ}$ linearly polarized beam on a polarizing wedge; the output will therefore be

$$
\mathbf{R}_{r}=\mathbf{J}_{\mathbf{P W}} \mathbf{R}_{+45^{\circ}}=\frac{1}{\sqrt{2}}\left(\begin{array}{c}
e^{-i \omega_{\|} x} \\
e^{-i \omega_{\perp} x}
\end{array}\right),
$$

The object beam goes through a half-wave plate to obtain a rotation of the original beam to provide the contrast bias, to go through the object. For the sake of simplicity, in what follows we will assume that at any stage it is possible to normalize the beam amplitude, which does not affect the end result. The Jones matrix for an object that rotates the input polarization by an angle $\phi(x, y)$ is given by

$$
\mathbf{J}_{O}=\left(\begin{array}{cc}
e^{-i \phi(x, y) / 2} & 0 \\
0 & e^{i \phi(x, y) / 2}
\end{array}\right) .
$$

The input beam on the object has a fixed polarization bias $2 \alpha$, which for our case is $2 \alpha=10^{\circ}$, and is given by

$$
\mathbf{I}_{o}=\left(\begin{array}{c}
e^{-i \alpha} \\
e^{i \alpha}
\end{array}\right),
$$


whereas the light leaving the object will be

$$
\mathbf{O}=\mathbf{J}_{O} \mathbf{I}_{o}=\left(\begin{array}{c}
e^{-i \phi(x, y) / 2-i \alpha} \\
e^{i \phi(x, y) / 2+i \alpha}
\end{array}\right) .
$$

The amplitude on the hologram plane will be given by

$$
\mathbf{A}_{H}=\mathbf{O}+\mathbf{R}_{\mathrm{ref}}=\frac{1}{\sqrt{2}}\left(\begin{array}{c}
e^{-i \omega_{\|} x}+e^{-i \phi(x, y) / 2-i \alpha} \\
e^{-i \omega_{\perp} x}+e^{i \phi(x, y) / 2+i \alpha}
\end{array}\right) .
$$

From the amplitude on the hologram plane we obtain the intensity, which is given by

$$
\begin{aligned}
& I_{H}=\frac{1}{2}\left(e^{i \omega_{\|} x}+e^{i \phi(x, y) / 2+i \alpha} \quad e^{i \omega_{\perp} x}+e^{-i \phi(x, y) / 2-i \alpha}\right) \\
& \times\left(\begin{array}{c}
e^{-i \omega_{\|} x}+e^{-i \phi(x, y) / 2-i \alpha} \\
e^{-i \omega_{\perp} x}+e^{i \phi(x, y) / 2+i \alpha}
\end{array}\right) \\
& =2+\frac{1}{2}\left[e^{i \phi(x, y) / 2+\alpha}\left(e^{-i \omega_{\|} x}+e^{-i \omega_{\perp} x}\right)\right. \\
& \left.+e^{-i \phi(x, y)-i \alpha}\left(e^{i \omega_{\|} x}+e^{i \omega_{\perp} x}\right)\right] .
\end{aligned}
$$

The transmission after processing, provided we are on the linear region of the response curve of the emulsion, is given by

$$
t(x, y)=t_{0}+\beta \tau I_{H},
$$

where $\beta$ is the slope of the response curve of the emulsion and $\tau$ the exposure time.

On reconstruction the hologram is illuminated with a beam given by $\mathbf{r}=r e^{-i \omega_{\|} x}$; we have

$$
\begin{aligned}
\mathbf{r} t(x, y)= & \mathbf{r} t_{0}+\mathbf{r} \beta \tau I_{H} \\
= & r e^{-i \omega_{\|} x}\left(t_{0}+4\right) \\
& +r e^{i \phi(x, y)+2 i \alpha}\left(e^{-i 2 \omega_{\|} x}+e^{-i\left(\omega_{\perp}+\omega_{\|}\right) x}\right) \\
& +r e^{-i \phi(x, y)-i 2 \alpha}\left(1+e^{i\left(\omega_{\perp}-\omega_{\|}\right) x}\right) .
\end{aligned}
$$

The last term of Eq. (9) shows that we have a set of parallel fringes, corresponding to the last term in parenthesis, modulated by the polarization rotation of the object. The fringe separation is given by the difference between $\omega_{\|}$and $\omega_{\perp}$, which is provided by the polarizing wedge. The angle of the reference beam to the object used in all our experiments is $12^{\circ}$, and the angular separation of the two polarizations that the polarizing wedge provides is $0^{\circ} 40^{\prime}$. The laser beam was filtered by using a spatial filter, resulting in a Gaussian beam of about $2 \mathrm{~cm}$, in which only the central $1 \mathrm{~cm}$ was used for the experiment. The intensity variation across the beam was less than $20 \%$.

The relative amplitude of each of the two reconstructed beams depends on the polarization angle between the object beam and each of the reference beams. For example, if at a particular point of the object the polarization is aligned with that of one of the reference beams, the reconstruction of the corresponding hologram will have maximum amplitude, whereas the reconstruction from the other hologram will have zero amplitude. Their amplitudes become equal for an angle of $45^{\circ}$. For an object in which there is no rotation a set of parallel fringes of constant contrast will be obtained, given that the object beam has an arbitrary bias of $2 \alpha=10^{\circ}$. The no-polarizationrotation area in the object provides a contrast bias from which it is possible to determine whether rotation is toward higher or lower polarization angles. The contrast bias is obtained by a $10^{\circ}$ polarization rotation of the object illumination beam. If the angle between the polarization of the object and one of the reference beams is $\theta$, the amplitude of the reconstructed beams will be

$$
A_{\|}=A \sin \theta
$$

$$
A_{\perp}=A \cos \theta
$$

and the corresponding intensities will be

$$
\begin{aligned}
& I_{\|}=A^{2} \sin ^{2} \theta, \\
& I_{\perp}=A^{2} \cos ^{2} \theta .
\end{aligned}
$$

As the two reconstructed beams have the same polarization state, because they are obtained from the same illuminating reconstructing beam, they interfere. The interference between the two reconstructed beams generates a set of parallel interference fringes. The fringe separation is controlled by the angle between the two beams. The fringe contrast [8] is given by

$$
V=\frac{I_{\max }-I_{\min }}{I_{\max }+I_{\min }}=2 \sqrt{\sin ^{2} \theta \cos ^{2} \theta} .
$$

As it turns out, the fringe contrast is dependent only on the polarization state. From Eq. (9) it is apparent that $\theta=\phi(x, y)+2 \alpha$.

\section{Experimental Procedure and Results}

In general, holographic emulsions have different responses that depend on the type of emulsion or the batch in which they were made. To obtain absolute measurements it is essential to perform a calibration. For calibration purposes an area of the object with a known polarization rotation is left; for all cases shown it is $10^{\circ}$. Figure 2 shows a reconstructed image in which the right-hand side has a rotation of $10^{\circ}$ to be used as calibration. Reconstructed images are recorded by using a CCD camera with $1024 \times$ 244 pixels and 16 bit resolution (SPH6 Apogee camera). An estimate of the minimum contrast from a CCD camera of a sine wave form can be obtained. In order to define a sine wave, at least six levels are required. For an 8 bit camera at mid-intensity the 


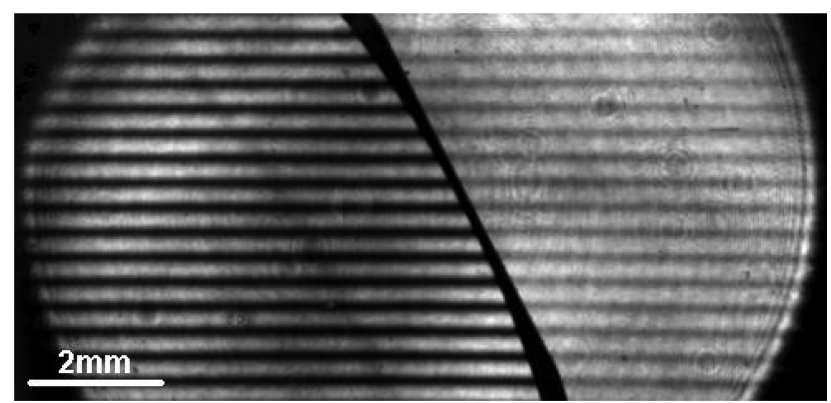

Fig. 2. Image of the reconstruction of a hologram. The reconstruction is divided into two zones; the right-hand side has a bias of $10^{\circ}$.

visibility will be $V_{8 \text { bit }}=6 / 128=0.047$. It is apparent that measuring a $1 \%$ change in contrast requires a camera with a minimum of 10 bits. From the reconstructed image, which is the interference pattern between the two images formed with each of the reference beams, an intensity profile is obtained, as is shown in Fig. 3. The two profiles are along lines perpendicular to the fringes in both the known polarization bias region and the unknown polarization region. From the profile extreme values are obtained. The values thus obtained are used to calculate the fringe contrast. The contrast is an average from around ten successive fringes. The contrast obtained from the known bias region $\left(10^{\circ}\right)$ is $V=$ 0.342 . From the values of contrast obtained in the known contrast area a calibration factor is obtained. The calibration factor is the ratio of 0.342 (contrast for the $10^{\circ}$ area) to the measured value of contrast on the $10^{\circ}$ area for the case in study. All values obtained from the image are subsequently multiplied by this sole calibration factor.

The procedure has been applied to several types of commercial holographic emulsion, which are detailed in Table 1 . In the foregoing discussion it was assumed that the emulsion has a linear response range and that the holograms were recorded on the linear range. We point out that the majority of tests were done with a solution physical developer [9], which has an extremely wide dynamic range, several others of magnitude of exposure energy. Further tests are required on other types of developer; however, as we apply a contrast bias, and the contrast variations are around this bias value, any developer ought to give comparable results.

As can be seen from Table 1 , there is a fairly broad dispersion of values of the normalizing factor for the different emulsions; however, after normalization

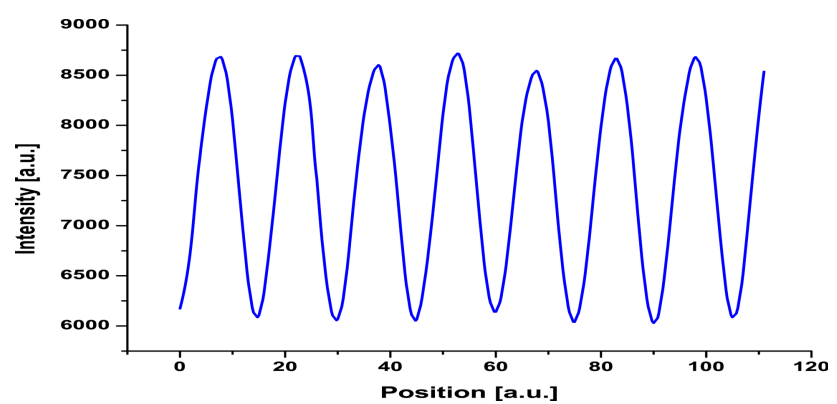

Fig. 3. (Color online) Typical line profile obtained from the image in Fig. $\underline{2}$.

the data points fall within a few percent of the same theoretical curve.

In Fig. 4 the theoretical curve for the fringe contrast is shown in conjunction with the normalized values for one of the emulsions tested. It is apparent that the measured values are well within the error bars of the measurement. Similar results were obtained for all emulsions tested.

Although it is possible to obtain two beams of perpendicular polarization with different angular directions using a combination of polarizing beam splitters and mirrors, the proposed scheme using a polarizing wedge is significantly simpler to align. Several of these schemes were tried, and always the presence of spurious fringes would render the reconstruction unusable. The optical alignment of the polarizing wedge setup is nearly as simple as a standard Fresnel hologram.

To assess the method described for measuring polarization rotation, a set of measurements of known samples was carried out. Two different solutions were used in a $1 \mathrm{~cm} \times 1 \mathrm{~cm}$ cross-section cuvette. A $25 \mathrm{~mm}$ diameter object beam was used, leaving an area around the cuvette that was used to obtain the calibration factor. Tartaric acid and saccharose were used as solute to make a solution in water. For both cases we used $41 \mathrm{~g}$ of solute for $100 \mathrm{~g}$ of solution. The results are given in Table 2 .

One of the advantages of the method proposed for polarization rotation is that it does have spatial resolution. To test this aspect of the method, in the same cuvette a solution with a gradient was obtained by slowly pouring two different concentrations. A solution of the same concentration used in the previous cases was poured into the cuvette, with pure water slowly poured on top of it. Initially some mixing does occur at the interface. It is necessary to wait around 20 min to obtain a useful gradient. The

Table 1. Tested Emulsions and Corresponding Typical Normalizing Factors Obtained

\begin{tabular}{lccrr}
\hline \multicolumn{1}{c}{ Emulsion } & Beam Ratio & Exposure Energy $\mu \mathrm{J} / \mathrm{cm}^{2}$ & Process & Normalizing Factor \\
\hline Ilford SP672T & $3: 1$ & 100 & CPA1/Fixer & 3.09 \\
Ilford SP695T & $3: 1$ & 100 & CPA1/Fixer & 10.83 \\
Agfa 10E56 & $3: 1$ & 100 & CPA1/Fixer & 5.56 \\
Ultimate 15 nm & $3: 1$ & Ultimate/Bleach & 2.87 \\
Ultimate 08 nm & $3: 1$ & 200 & Ultimate/Bleach & 1.83 \\
Fujifilm F HL & $3: 1$ & 150 & CPA1/Fixer & 4.76 \\
\hline
\end{tabular}




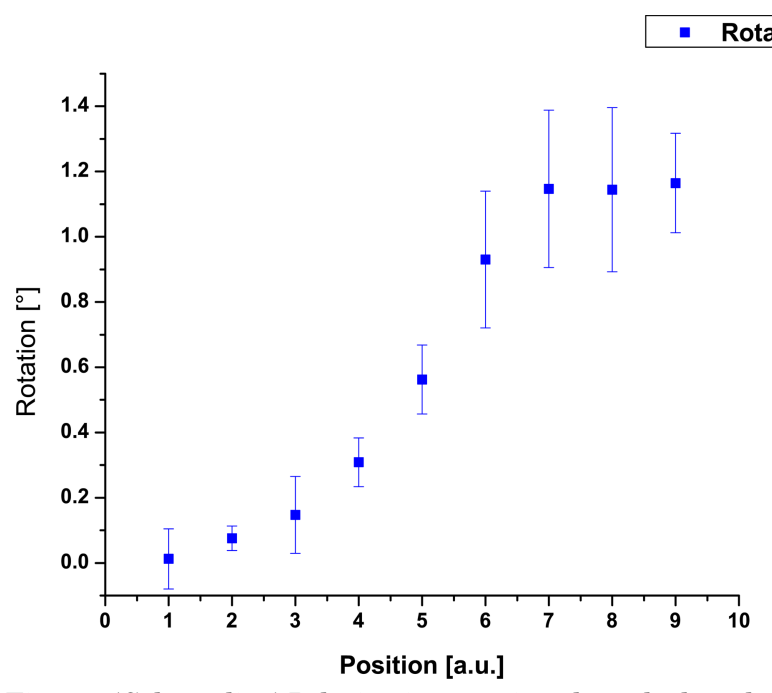

Fig. 4. (Color online) Polarization rotation along the length of the cell in which a solution with a gradient concentration of tartaric acid was established.

results obtained are shown in Fig. 5 , which demonstrate the capability of the method to obtain spatial information. Each division is approximately $1 \mathrm{~mm}$ on the actual object.

\section{Conclusions}

A method suitable for measuring the polarization rotation of coherent light has been proposed, which is a two-stage process. Initially two holograms are recorded simultaneously on the same holographic emulsion, using reference beams that have mutually perpendicular polarizations. The two reconstructed images from the hologram interfere to produce a set of parallel fringes; the rotation measurements are obtained from the fringe contrast. This method is well suited for performing two-dimensional measurements with spatial resolution. This method is particularly well suited to transient phenomena, as in dense plasma studies. For this type of study, a pulsed laser can obtain the polarogram, to be analyzed at a later stage. This type of study is not feasible using [6], as it requires the acquisition of several images to obtain a measurement. To provide a

Table 2. Rotation Values for Solutions (41 g Solute, $100 \mathrm{~g}$ Solution)

\begin{tabular}{cccc}
\hline $\begin{array}{c}\text { Active } \\
\text { Media }\end{array}$ & $\begin{array}{c}\text { Expected } \\
\text { Rotation }\end{array}$ & $\begin{array}{c}\text { Measured } \\
\text { Rotation }\end{array}$ & Error \\
\hline Tartaric acid & $1^{\circ}$ & $1.043^{\circ} \pm 0.288^{\circ}$ & $4.3 \%$ \\
Saccharose & $7.4^{\circ}$ & $7.161^{\circ} \pm 0.346^{\circ}$ & $3.2 \%$ \\
\hline
\end{tabular}

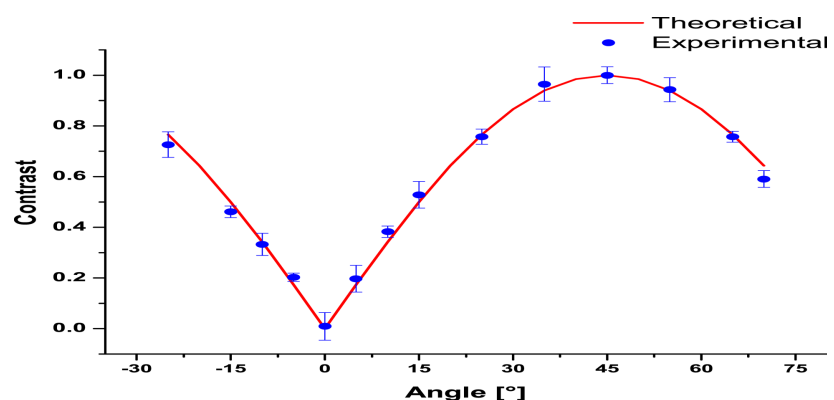

Fig. 5. (Color online) Comparison of normalized measured values for a typical case against the theoretical curve.

calibrating factor for the holographic emulsion used it is necessary to have an area with known rotation. The values obtained from the measurements have errors that are less than $5 \%$, and spatial variation can also be obtained. One of the interesting advantages of the present measurement technique is that noncoherent light does not affect the results obtained. This is because only the coherent light from the laser contributes to the hologram formation; any incoherent light will not show up in the reconstruction of the holograms.

The authors gratefully acknowledge funding under Fondo Nacional de Desarrollo Científico y Tecnológico (FONDECYT) grant 1030958.

\section{References}

1. A. W. Lohmann, "Reconstruction of vectorial wavefronts," Appl. Opt. 4, 1667-1668 (1965).

2. K. Gasvik, "Holographic reconstruction of the state of polarization," J. Mod. Opt. 22, 189-206 (1975).

3. H. Kubo and R. Nagata, "Further consideration of photoelasticity using polarization holography," J. Mod. Opt. 23, 519528 (1976)

4. D. Garvanska, "Polarization holography applied to detection of shape deviations of metal surfaces," J. Opt. 12, 201206 (1971).

5. T. Todorov, L. Nikolova, and N. Tomova, "Polarization holography. 1: new high-efficiency organic material with reversible photoinduced birefringence," Appl. Opt. 23 4309-4312 (1984).

6. R. J. Wijngaarden, K. Heeck, M. Welling, R. Limburg, M. Pannetier, K. van Zetten, V. L. G. Roorda, and A. R. Voorwinden, "Fast imaging polarimeter for magneto-optical investigations", Rev. Sci. Instrum. 72, 2661-2664 (2001).

7. M. Born and E. Wolf, Principles of Optics, 4th ed. (Pergamon, 1970).

8. W. T. Welford, Optics, Vol. 14 of Oxford Physics Series (Oxford University Press, 1976).

9. R. Aliaga, H. Chuaqui, and P. Pedraza, "Achival and wide exposure latitude process for holography," Appl. Opt. 29, 28612863 (1990). 